\title{
Transarterial glue embolization using a balloon flow arrest technique for arteriovenous fistula
}

\author{
Makoto SAKAMOTO ${ }^{1)}$ Mitsutoshi KADOWAKI ${ }^{1)}$ Minoru MIZUSHIMA ${ }^{1)}$ Takashi WATANABE ${ }^{1)}$ \\ Hirochika TAKEUCHI ${ }^{2)}$ Minoru OHTAKE ${ }^{3)}$ Yukiya INOUE3) \\ 1) Department of Neurosurgery, Tottori University \\ 2) Department of Neurosurgery, Nojima Hospital \\ 3) Department of Neurosurgery, Hamada Medical Center
}

\begin{abstract}
Objective: Transarterial embolization (TAE) using liquid embolic material is adopted in the treatment of dural arteriovenous fistulas (DAVFs) with direct cortical venous drainage or in patients in whom transvenous access is limited. Arresting flow by wedging a microcatheter tip into a main feeding vessel is important to achieve complete obliteration of DAVF with TAE. The Masamune catheter is a double lumen microcatheter with a silicone balloon at its distal end, characterized by the shortness of its catheter tip to the distal balloon end. Two cases of DAVF treated by TAE with the balloon flow arrest technique using a Masamune balloon microcatheter are reported.

Case Report: Case 1, a 50-year-old man, was incidentally diagnosed with a tentorial DAVF with direct cortical venous drainage. An inflated Masamune balloon catheter was wedged in the occipital artery, and trans-arterial embolization was performed under a flow-arrested condition. As preparative embolization of the other minor feeding arteries had not been performed, complete obliteration of DAVF was not achieved by competing flow.

Case 2, a 64-year-old man, was diagnosed with a left transverse-sigmoid DAVF on angiogram. The DAVF was completely obliterated with the balloon flow arrest technique using liquid embolic material.

Conclusion: The Masamune catheter can easily achieve an artificial flow-arrest condition by inflation of its distal balloon to prevent fragmentation of liquid embolic material. In conjunction with preparative embolization of minor feeding vessels, curative embolization may be achieved from a main feeder using this catheter.
\end{abstract}

Key Words

arteriovenous fistula, balloon microcatheter, transarterial emobolization

<Correspondence Address : Sakamoto M, 36-1, Nishicho, Yonago, Tottori, JapanＥ-mail: sakamako@grape.med.tottori-u.ac.jp>

(Received March 28, 2008 : Accepted May 27, 2008)

\section{Introduction}

Treatment options for intracranial dural arteriovenous fistulas (DAVFs) include direct surgery, endovascular treatment, including transarterial and/or transvenous embolization, radiosurgery and combinations of these treatments ${ }^{4,7)}$. To achieve a complete cure and to eliminate neurological complications, the arteriovenous shunt must be completely closed during every treatment procedure, and normal cerebral venous drainage must be preserved. Except for anterior cranial fossa, tentorial and craniocervical junction DAVFs, endovascular procedures, particularly transvenous embolization, have proven to be useful in treating many DAVFs, obliterating the fistula by sacrificing the affected sinus ${ }^{10)}$. However, in patients harboring DAVFs with direct cortical venous drainage or for whom transvenous access is limited because of venous sinus thrombosis or obstruction, transvenous embolization may be impossible, and transarterial embolization must be considered ${ }^{9)}$. Effective TAE of a DAVF requires penetration of a durable embolic agent into the fistulous connection with a preservation of normal venous drainage. Some authors emphasize techniques that involve wedging a microcatheter into the main feeding artery to inject a diluted mixture of $\mathrm{N}$-butyl cyanoacrylate (NBCA) glue and iodized oil. Others also emphasize the importance of preparatory devascularization of other minor feeding arteries by embolization to avoid fragmentation of the glue column by competing inflows ${ }^{3,9)}$.

The wedge technique requires navigation to a sufficiently distal segment so that the diameter of the feeding artery must match that of the microcatheter tip. Such positioning of a microcatheter is not always possible in the large caliber, tortuous conduits that typically supply DAVFs.

The Masamune catheter (Fuji Systems, Tokyo, Japan) is a 

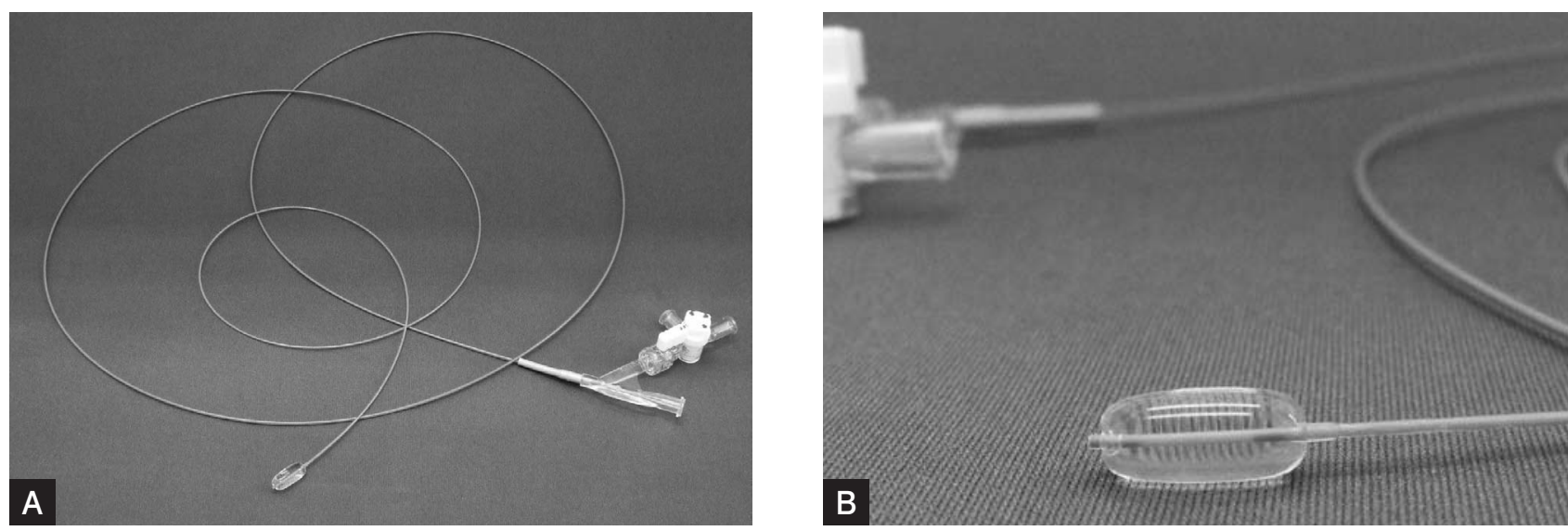

Fig. 1 The Masamune catheter (Fuji Systems, Tokyo, Japan) is a double lumen microcatheter with a silicone balloon at its distal end (A), characterized by the shortness of its catheter tip to the distal balloon end (B).
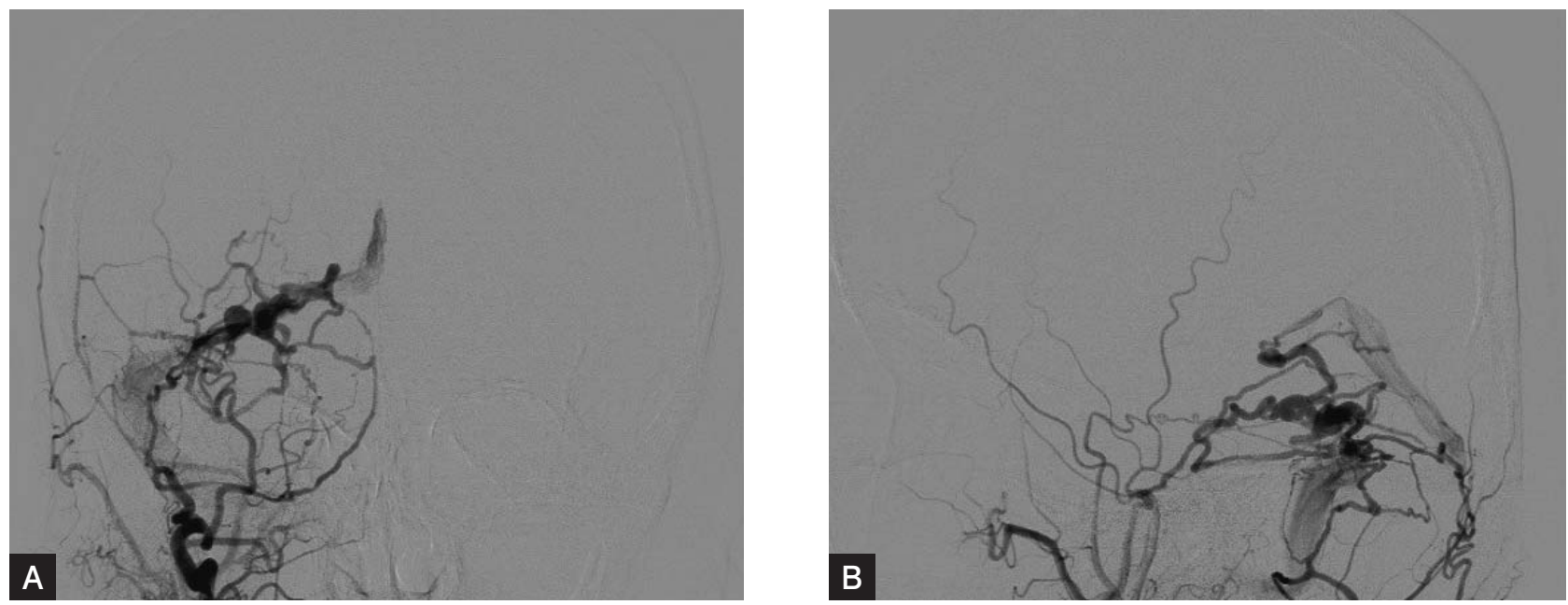

Fig. 2 Right external carotid angiogram, anterior-posterior view, indicates a tentorial DAVF mainly supplied by the right occipital artery (A). Marked retrograde venous drainage is visualized. Right external carotid angiogram, lateral view, indicates tentorial DAVF supplied mainly by branches of the external carotid artery, and drained via the petrosal vein into the basal vein of Rosenthal (B).

double lumen microcatheter with a silicone balloon at its distal end (Fig. 1A), characterized by the shortness of its catheter tip to the distal balloon end (Fig. 1B). The distal ends of Masamune catheters can always be artificially wedged in place by inflation of the balloon leading to a condition of continuous flow arrest, so that fragmentation of liquified embolic material is prevented.

In this report, a new technique that improves the distal distribution of NBCA glue by balloon wedge injection using a Masamune balloon microcatheter is described.

\section{Case Presentation}

\section{Case 1}

A 50-year-old male patient was incidentally diagnosed with a tentorial DAVF with cortical venous drainage by magnetic resonance angiogram. Cerebral angiography demonstrated a
DAVF with arterial supply from branches of the middle meningeal arteries, the stylomastoid branch of the occipital artery, the ascending pharyngeal artery, and a branch of the right posterior cerebral artery (Fig. 2A, B). Venous drainage was via the petrosal vein to the basal vein of Rosenthal and via the superior petrosal sinus to the right sigmoid sinus. The patient was completely asymptomatic, therefore it was decided to perform endovascular obliteration of the fistula using liquid embolic material. Prior to treatment, informed consent was obtained from the patient. The main feeder, a branch of the occipital artery, had a large caliber and was considerably tortuous, therefore it would likely be difficult to advance distally and wedge a microcatheter. First, the Masamune balloon microcatheter was advanced over a microguidewire (Transend Ex floppy 0.010 inch, Boston Scientific, Natick, MA, USA) into the occipital artery mainly supplying the 

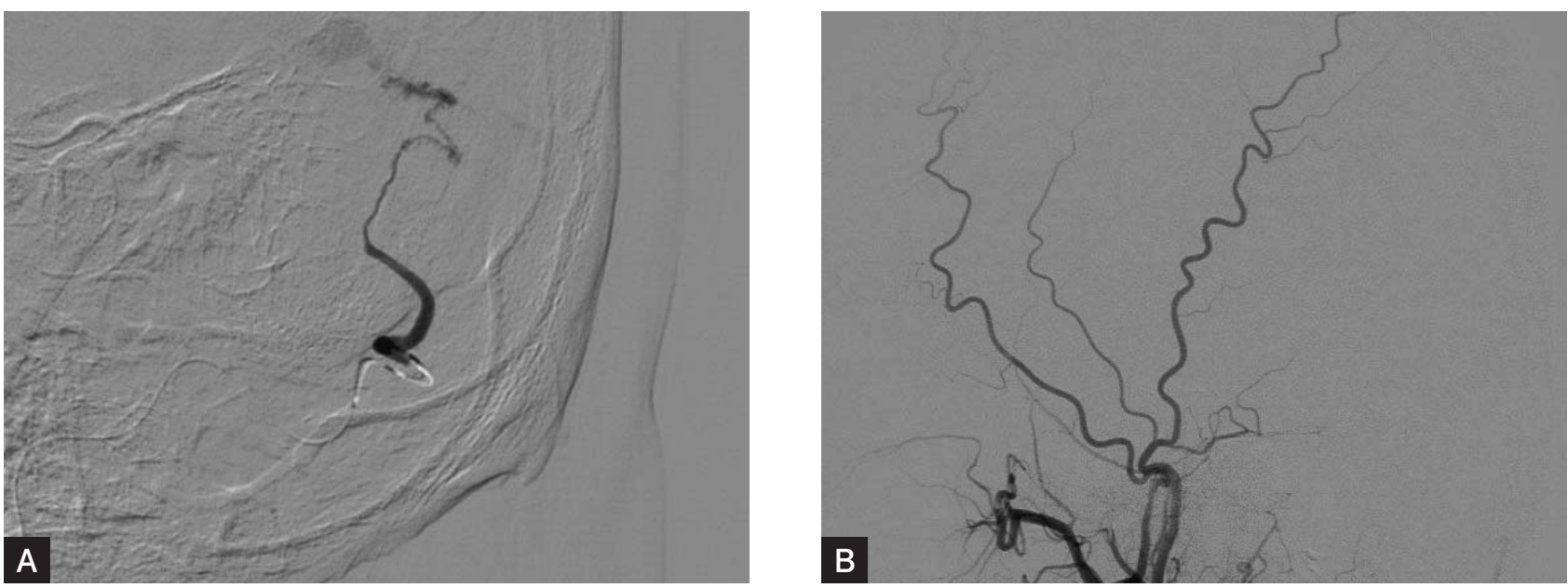

Fig. 3 Superselective angiogram via an inflated Masamune catheter indicates a continuing pathway between the feeding artery and draining vein (A). Postoperative external carotid angiogram, lateral view (B) indicates disappearance of an arterio-venous shunt arising from branches of the external carotid artery.

DAVF. The tip of the microcatheter was introduced into the occipital artery and a superselective angiogram was performed. Prevented by rapid flow, it was difficult to interpret the flow dynamics from the superselective angiogram without balloon inflation. However, a superselective angiogram under conditions in which the microcatheter was artificially wedged in place by inflating the balloon attached to the tip of the microcatheter enabled visualization of the angiographic architecture of the lesion (Fig. 3A), i.e. the feeding arteries, fistula point, and draining veins could all be visualized. At first, transarterial embolization was performed under the flow-arrest condition by inflating the balloon microcatheter. The guidewire lumen of the Masamune catheter that had been rinsed with $4 \mathrm{~mL}$ of $5 \%$ dextrose in water, then $0.6 \mathrm{~mL}$ of $20 \% \mathrm{NBCA} /$ lipiodol mixture was advanced slowly without fragmentation of liquid embolic material. Immediately after the NBCA had penetrated a fistula of the DAVF, injection of NBCA was stopped, then the silicon balloon of the Masamune catheter was slowly deflated. As NBCA does not adhere to the silicon, it is not necessary to remove the Masamune catheter rapidly. As it was supplied by other minor feeding arteries, the fistula was not effectively embolized. Subsequently, transarterial embolization via MMA and the ascending pharyngeal artery was performed using a microcatheter without a balloon. A right external carotid angiogram indicated disappearance of the DAVF (Fig. 3B), however a minor feeder via a branch of the posterior cerebral artery (PCA) remained. Embolization of the distal PCA was abandoned, as shunt flow had apparently reduced and embolization of the PCA had potential to induce neurological complications, such as cerebral stroke. Stereotactic radiosurgery was applied to the patient, and the lesion disappeared 1 year later.

\section{Case 2}

A 64-year-old male presented with consciousness disturbance induced by left temporal lobe subcortical hemorrhage and emergent evacuation of a hematoma was performed without detailed angiographic evaluation at a former hospital. Postoperative angiogram indicated transverse-sigmoid DAVF with retrograde leptomeningeal venous drainage, therefore the patient was referred to our hospital for endovascular treatment. Prior to treatment, informed consent was obtained from the patient. A 6 Fr guiding catheter was placed into the left occipital artery under local anesthesia. An angiogram indicated a transverse sigmoid DAVF mainly supplied from a branch of the occipital artery and drained via an isolated sinus to deep veins (Fig. 4A, B). Initially, transvenous embolization was applied because of its high curability rate and low recanalization rate. A 0.035 -inch guidewire and a 0.014-inch microguidewire could advance into the isolated transverse sinus via the obstructed sigmoid sinus, however a microcatheter could not pass through the obstructed sigmoid sinus. Transvenous embolization was abandoned, and transarterial embolization applied using the balloon flow arrest technique. A Masamune catheter was advanced into the occipital artery over the 0.010-inch microguidewire, however a superselective angiogram using the Masamune catheter without inflation of the balloon could not visualize the detailed angiographic architecture of the lesion, due to its high-flow nature. Using 

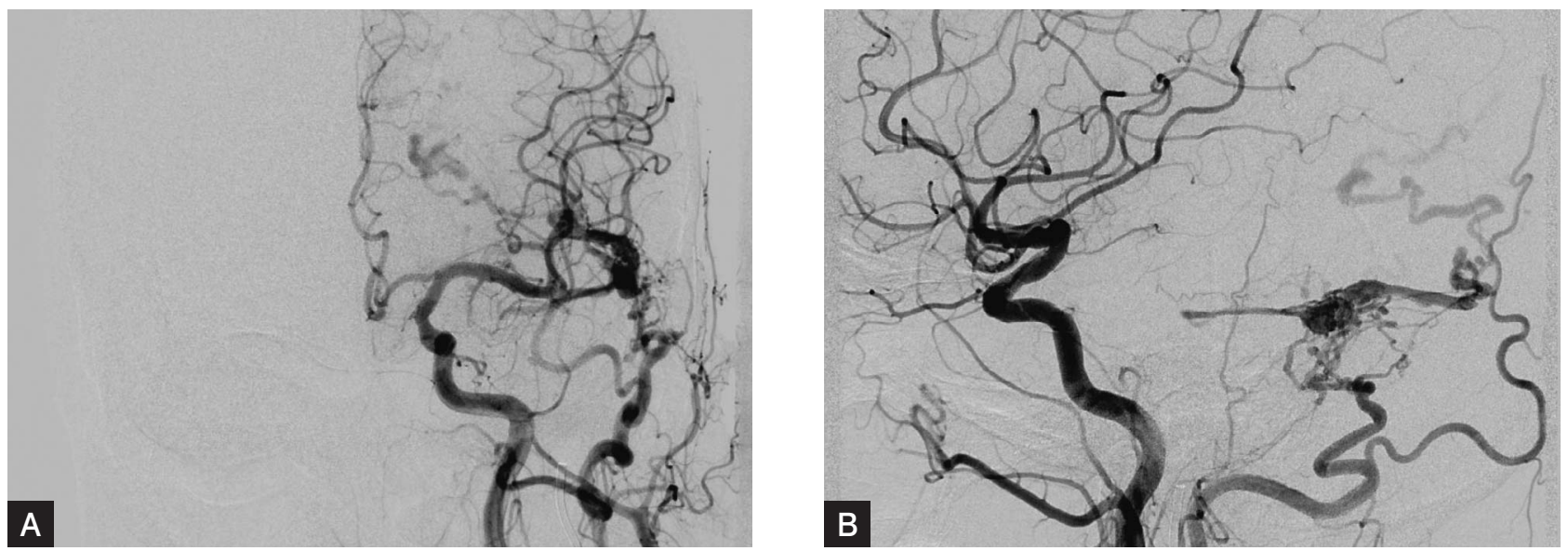

Fig. 4 Pre-embolization common carotid angiogram, antero-posterior view (A) and lateral view (B), indicates a transverse-sigmoid DAVF mainly supplied by the occipital artery. The DAVF is drained via an isolated transverse sinus to deep veins.

the balloon flow arrest technique superselective angiogram however enabled visualization of the fistulous component and deep retrograde venous drainage (Fig. 5A). An angiogram via a guiding catheter inflating the balloon of a Masamune catheter indicated that there were few other minor feeding arteries contributing to the lesion (Fig. 5B). Under the flowarrest condition achieved by inflating the balloon of the Masamune catheter (Fig. 5C), transarterial embolization using $0.5 \mathrm{~mL}$ of $20 \% \mathrm{NBCA} /$ lipiodol mixture was performed in the same way as in case 1 . Complete obliteration of the DAVF was achieved using this balloon wedge injection technique, and no new neurological deficits were observed after the embolization (Fig. 6A, B).

\section{Discussion}

A new technique that improves the distal distribution of NBCA glue by balloon wedge injection of liquid embolic material using a Masumane balloon microcatheter is presented. The distal catheter tip of a Masamune catheter can easily be wedged in place in a feeding artery by inflating its distal balloon. Distal penetration of liquid embolic material is then enabled by arresting the flow of the main feeding artery, and complete obliteration of DAVF may be achieved.

To achieve a complete cure and to eliminate neurological complications, the arteriovenous shunt must be completely closed in TVE, and normal cerebral venous drainage must be preserved. However, in patients harboring DAVFs with direct cortical venous drainage or in whom transvenous access is limited because of venous sinus thrombosis or obstruction, transvenous embolization may be impossible and transarterial embolization must be considered. Effective TAE of a DAVF requires penetration of a durable embolic agent into the fistulous connection with a preservation of normal venous drainage systems ${ }^{9}$. Platinum coils often cannot traverse the tortuous vessels, leading to proximal occlusion and recruitment of collateral flow. Polyvinyl alcohol (PVA) particles may achieve better penetration, but their effects are temporary, often leading to recanalization ${ }^{4)}$. NBCA glue, an adhesive liquid embolic material, polymerizes on contact with ionic solutions, such as contrast medium, saline or blood, distributes more distally than platinum coils, and its recanalization rate is considerably low. High-concentration NBCA can cause a failure of DAVF treatment, due to its tendency to produce proximal occlusion, so use of a lowconcentration NBCA mixture for DAVF treatment is widely accepted when aiming for a complete cure ${ }^{6}$. Lipiodol acts as a buffering agent, which delays the contact between NBCA and the blood, thereby indirectly delaying the initiation of NBCA polymerization. However, under high-flow conditions, low-concentration NBCA may be easily fragmented and early polymerization of NBCA will occur in distal draining veins. In such situations, NBCA may not be able to reach the lesion itself, rendering the embolization ineffective. Conversely, deposition of embolic agents distal to the fistula may result in venous infarction or hemodynamic alterations that promote hemorrhage ${ }^{2,5}$. To prevent these hazardous complications, some authors emphasize techniques that involve wedging a microcatheter into the main feeding artery to inject a diluted mixture of NBCA/lipiodol mixture, and preparatory devascularization of other minor feeding arteries by embolization to avoid fragmentation of the glue column by competing inflows ${ }^{3,49)}$. When the microcatheter is wedged 

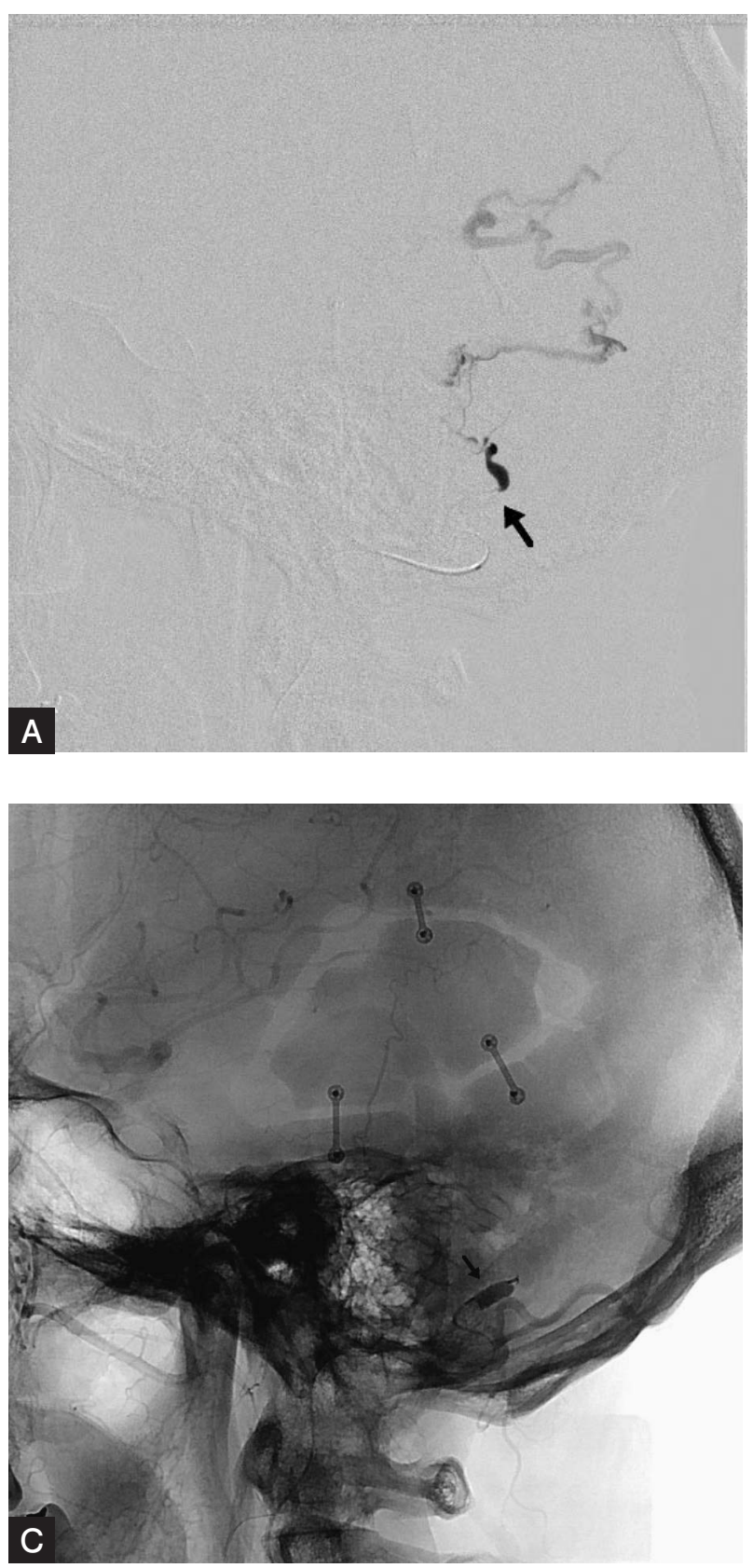

within the targeted artery and causes flow arrest in its distal distribution, injecting $5 \%$ dextrose in water through the microcatheter provides an adequate "stagnant" non-ionic environment that allows excellent distal NBCA progression. However, the wedge technique requires navigation to a sufficiently distal segment so as to match the diameter of the feeding artery and the microcatheter tip. Also, repeated attempts at wedging a microcatheter in place may result in adverse events, such as an arterial dissection that may temporarily preclude subsequent embolization. Such positioning of the microcatheter is not always possible in the

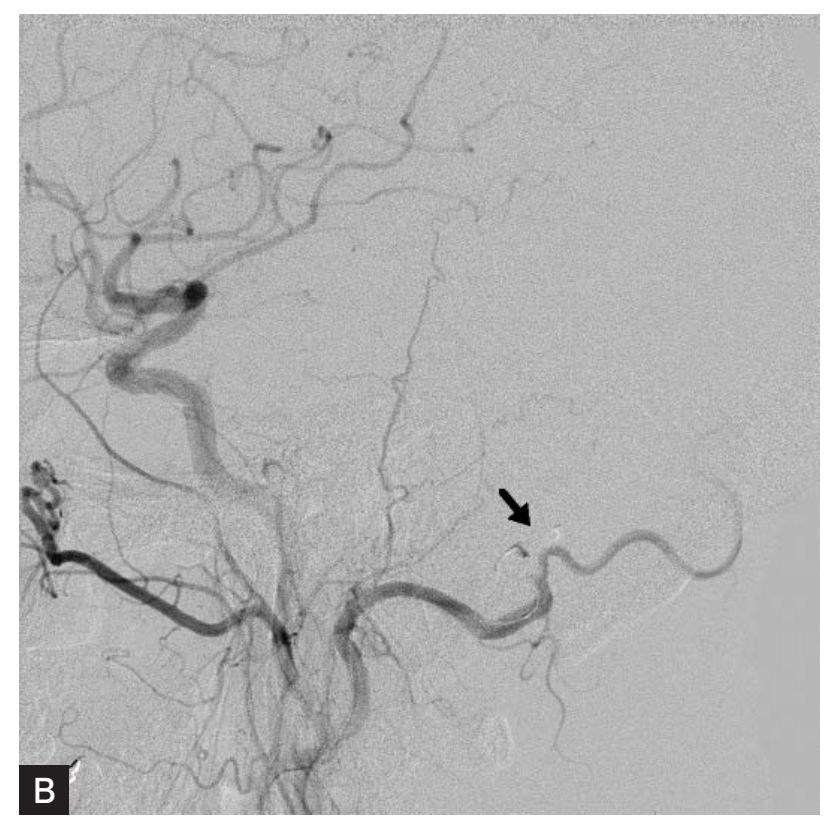

Fig. 5 Superselective angiogram via an inflated Masamune catheter indicates a continuing pathway between the feeding artery and draining vein. Despite the comparatively large caliber of the occipital artery, flow arrest is achieved by inflating the balloon of the Masamune catheter. Detailed angiographic architecture and circulation of blood flow are demonstrated clearly (A, arrow indicates the tip of the Masamune catheter).

Common carotid angiogram under the condition of an inflated Masamune catheter indicates that there are few minor feeding arteries in addition to the main feeding artery (B). Arrow indicates the balloon of the Masamune catheter.

Masamune catheter is wedged in place by inflating its distal balloon (arrow) and the shortness of its catheter tip to the distal balloon end is indicated on a non-subtracted angiogram (C).

large caliber, tortuous conduits that typically supply DAVFs.

Masamune catheters can always be wedged in place at their distal ends by inflation of the balloon without advancing the microcatheter more distally. As a result, the main feeding vessel will be in a condition of artificial continuous flow arrest, without any relationship to vessel caliber, so that fragmentation of liquid embolic material is prevented. Operators can easily control flow speed of liquid embolic material and volume of the low-concentration NBCA by regulating the injection force without fragmentation of the NBCA. Additionally, to perform an angiogram from a guiding 

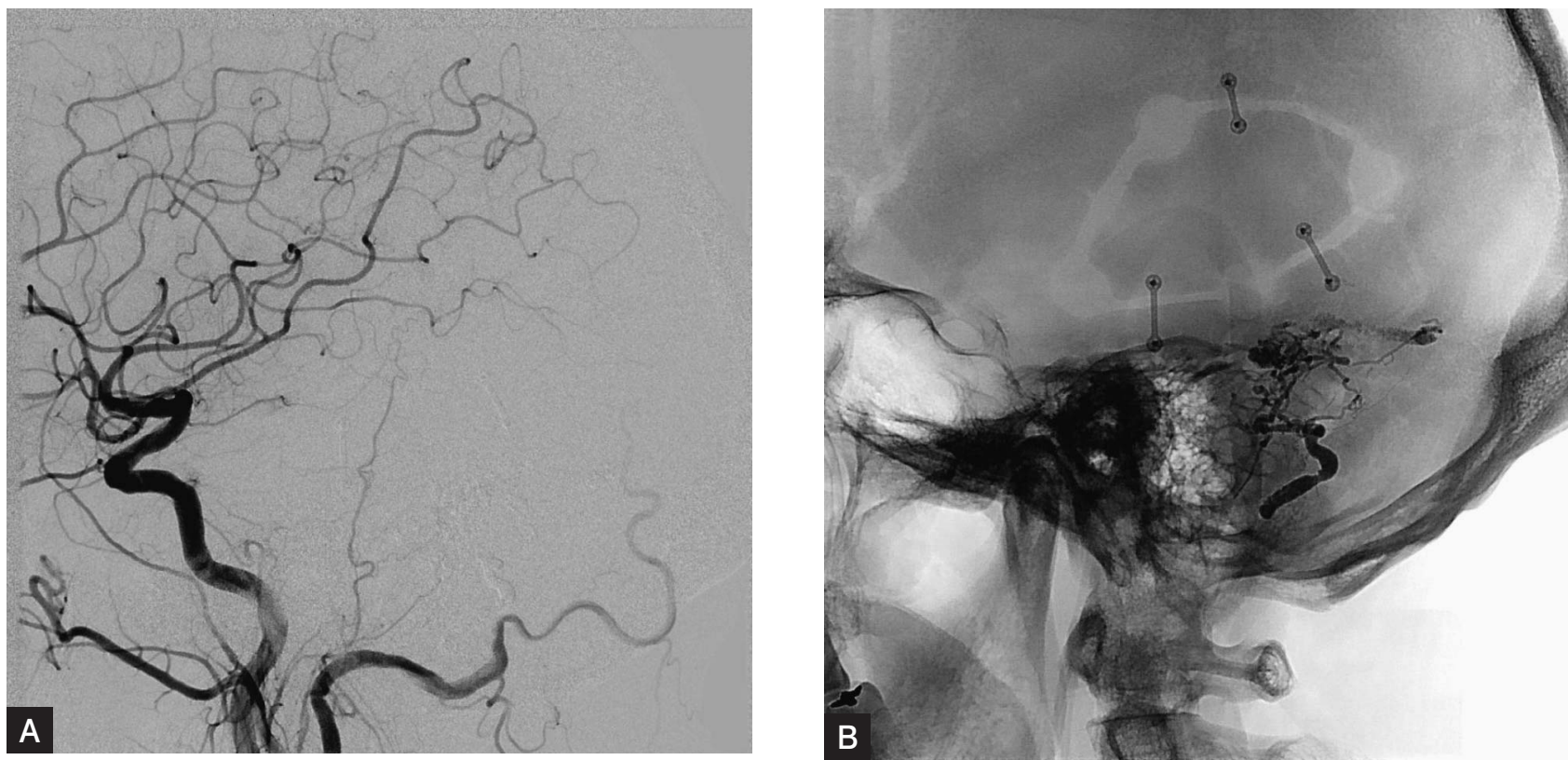

Fig. 6 Post-embolization left common carotid angiogram, lateral view, shows complete resolution of the DAVF (A). A non-subtracted angiogram shows distal penetration of NBCA into the draining vein (B).

catheter while inflating the distal balloon of the Masamune catheter, presence of minor feeding vessels other than the main feeding artery can be confirmed preoperatively. Furthermore, adhesion to liquid embolic material is prevented as the catheter tip is very short and the silicon balloon will not adhere on contact with adhesive liquid embolic material such as NBCA.

For case 1, complete devascularization was not achieved in TAE, though NBCA distributed to a fairly distal segment of the tortuous feeding artery. Many minor feeding arteries supplied the DAVF in case 1. The main feeder was embolized initially, therefore complete obliteration using TAE was not achieved due to competing flow from other minor feeding arteries. The lesson from case 1 is, even when a wedged microcatheter position is achieved, early polymerization within the feeder proximal to the actual arteriovenous shunt can still occur, resulting in suboptimal embolization. This early polymerization is probably related to the plexiform nature of the arterial vascularization of DAVFs: even when the microcatheter tip occludes one feeder, other arterial contributors may still contribute to flow. In a similar fashion, some reports have emphasized the importance of preparatory embolization of accessory pedicles with the flow-related approach to decrease competing inflow and fragmentation of the glue column.

Disadvantages of this balloon wedge injection technique are the flexibility, trackability and selectability of the Masamune catheter, which are inferior to those of single lumen microcatheters and flow-guided microcatheters. The distal end of the Masamune catheter is hard, because of its double lumen construction, so the flexibility of Masamune catheter is the worst of all available microcatheters for cerebral embolization. Also, its guidewire lumen is narrow, and only compatible with 0.010-inch microguidewires, so the trackability of the Masamune catheter is inferior to other single lumen microcatheters. In addition, the shortness of its distal end prevents a preparative steam shape, so the selectability of branched arteries is not satisfactory. Small and less tortuous feeding arteries should be wedged using a single lumen microcatheter, and the Masamune catheter should be applied to large caliber, tortuous conduits that typically supply DAVFs.

Possible complications associated with transarterial NBCA embolization of DAVFs, especially in the wedge condition, may include ischemic cranial nerve palsies, trans-collateral embolization into normal cerebral arteries, and systemic venous embolization with $\mathrm{NBCA}^{9)}$. Due to its lesser flexibility, trackability and selectability, a Masamune catheter may not advance into a feeding artery distal enough to ignore dangerous anastomoses, therefore embolization using a Masamune catheter may fail. To avoid these aggravating complications, the angiographic architecture of DAVFs must be evaluated preoperatively, to determine the location of dangerous anastomoses between feeding arteries and normal 
vessels. A superselective angiogram in the artificially wedged condition may improve understanding of the angiographic architecture of high-flow DAVFs and help to decide the concentration of the NBCA/lipiodol mixture by evaluating the circulation between the feeding artery and the draining vein. Provocative testing using lidocaine under balloon wedge condition may also help to avoid neurological complications.

Recently, some have reported coaxial injection of $5 \%$ dextrose in water through the guiding catheter, results in improved penetration of $\mathrm{NBCA}^{1,8}$. In this technique, glue is injected through the microcatheter, and a column of $5 \%$ dextrose in water is pushed manually through the guide catheter lumen to propel the glue forward. Combinations of the wedge technique and this coaxial injection of $5 \%$ dextrose in water through the guiding catheter may further improve the penetration of NBCA.

\section{Conclusion}

The balloon wedge injection technique ameliorated distal penetration of NBCA and achieved good clinical results without neurological complications. Flow arrest condition can easily be obtained by wedging a Masamune balloon microcatheter in place within a vessel. Detailed evaluation of the angiographic architecture of DAVFs and knowledge of normal vascular anastomoses are indispensable for successful treatment. Preparatory devascularization of other minor feeders is required for complete resolution of DAVFs by TAE.

\section{References}

1) Amar AP, Teitelbaum GP, Larsen DW: A novel technique and new grading scale for the embolization of cerebral vascular malformations. Neurosurgery 59 (suppl 3):S158-162, 2006.
2 ) Cognard C, Gobin YP, Pierot L, et al: Cerebral dural arteriovenous fistulas: clinical and angiographic correlation with a revised classification of venous drainage. Radiology 194: 671-680, 1995.

3 ) Iizuka Y, Maehara T, Hishii M, et al: Successful transarterial glue embolisation by wedged technique for a tentorial dural arteriovenous fistula presenting with a conjunctival injection. Neuroradiology 43:677-679, 2001.

4 ) Kiyosue H, Hori Y, Okahara M, et al: Treatment of intracranial dural arteriovenous fistulas: current strategies based on location and hemodynamics, and alternative techniques of transcatheter embolization. Radiographics 24:1637-1653, 2004.

5 ) Kurata A, Miyasaka Y, Yoshida T, et al. Venous ischemia caused by dural arteriovenous malformation. J Neurosurg 80:552-555, 1994.

6 ) Liu HM, Huang YC, Wang YH, et al: Transarterial embolisation of complex cavernous sinus dural arteriovenous fistulae with low-concentration cyanoacrylate. Neuroradiology 42:766-770, 2000.

7 ) Lucas CP, Zabramski JM, Spetzler RF, et al: Treatment for intracranial dural arteriovenous malformations: a meta-analysis from the English language literature. Neurosurgery 40:1119-1132, 1997.

8 ) Moore C, Murphy K, Gailloud P: Improved distal distribution of n-butyl cyanoacrylate glue by simultaneous injection of dextrose $5 \%$ through the guiding catheter: technical note. Neuroradiology 48:327-332, 2006

9 ) Nelson PK, Russell SM, Woo HH, et al. Use of a wedged microcatheter for curative transarterial embolization of complex intracranial dural arteriovenous fistulas: indications, endovascular technique, and outcome in 21 patients. J Neurosurg 98:498-506, 2003.

10) Roy D, Raymond J: The role of transvenous embolization in the treatment of intracranial dural arteriovenous fistulas. Neurosurgery 40:1133-1144, 1997. 